\title{
Stochastic modelling of tear behaviour of coated fabrics
}

\author{
Wen Zhong ${ }^{1}$, Ning Pan ${ }^{1,3}$ and David Lukas ${ }^{2}$ \\ ${ }^{1}$ Division of Textiles and Clothing, Department of Biological and Agricultural Engineering, \\ 2 Technical University of Liberec, Liberec, Czech Republic \\ E-mail: npan@ucdavis.edu
}

Received 30 July 2003

Published 16 February 2004

Online at stacks.iop.org/MSMSE/12/293 (DOI: 10.1088/0965-0393/12/2/010)

\begin{abstract}
A stochastic approach, using the Ising model combined with the Monte Carlo simulation, is employed to study the phenomenon of tongue tear failure in coated fabrics. The complicated mechanisms involved can be realistically simulated with a relatively simple algorithm. The important factors, especially the effects of the interphase between the coating and the fabric, and the stretched part of the material at the crack front (the del-zone) can be represented by corresponding coefficients in the Hamiltonian expression of the system. The minimization of the system Hamiltonian yields the most likely new steps for crack propagation, while the Monte Carlo method is used to select the one that will actually occur, reflecting the stochastic nature in the behaviour of real systems, indicating the usefulness of this approach in studies of similar interfacial phenomena. However, this model like many others needs to be calibrated based on data from a real system for quantitative and accurate predictions.
\end{abstract}

\section{Introduction}

As a flexible composite material, the coated fabric is formed by applying a coating agent to either one or both sides of a fabric substrate, thus creating an interphase between them. In other words, the interphase is a mixture formed during the coating process as the coating agent penetrates into the fabric substrate.

Although the interphase is composed of the fabric substrate and the coating agent, it may possess properties different from the original ex situ properties of either constituent, just like in ordinary composites $[1,2]$. This interphase may exacerbate the interactions between the fabric substrate and the coating agent that comprise the coated fabric.

An interphase is well known to contribute significantly to the mechanical properties of a composite material, and mathematical analysis of the interfacial behaviour has been attempted extensively. Nevertheless, the inclusion of an interphase region has been neglected in many

3 Author to whom any correspondence should be addressed. 


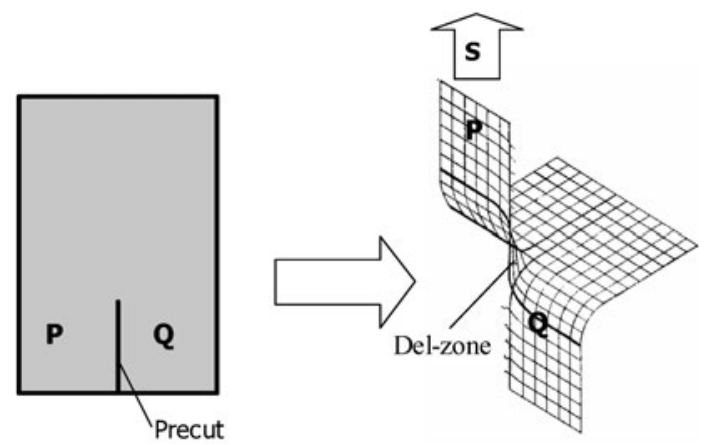

Figure 1. Tongue tear test.

such analyses, such as in a shear-lag type analysis [3], in a Bessel-Fourier series stress analysis [4], and in a variational mechanics model [5], to name just a few. Nairn and Liu [6] considered such an interphase to be analogous to an imperfect interface whose property is a function of its bond quality. Nevertheless, even in their model, the interphase was still treated as having zero thickness. Other authors [7, 8] discussed theoretically the effects of interphase of certain thickness, yet it is considered more realistic to treat composites as three-component systems with the interphase as the third component [9]. Using the method of unit cells, Gardner et al [1] and Low et al [2] formulated a micromechanical model to characterize the three-phase composites that contain either a homogeneous interphase [1] or a heterogeneous one with property gradients [9] in the thickness direction. Applying fragmentation tests, Hayes et al $[10,11]$ assessed the effect of interphase on the load transfer from matrix to a single fibre. Usually, these models involve complex FEM analysis, requiring a lot of computing resources when dealing with problems involving complex structures and intricate geometry, or nonlinear phenomena.

On the other hand, experimental investigations on composite interphase have shed considerably light on the issue [12]. For instance, atomic force microscopy (AFM) has been used to assess interphase characteristics, showing either constant, or graded properties across the interphase region [13]. Such work validates micromechanical analyses intended to model the interphase either as a third homogeneous phase with properties averaged over those of the other two components, or as a heterogeneous phase possessing graded properties [14]. In the latter case, which has received more attention, the composite materials are sometimes referred to as functionally graded materials (FGM) [15]. In this paper, the interphase between the fabric substrate and the coating agent is also regarded as a phase with graded properties.

Increased application of coated fabrics has been demanding a better understanding of the behaviour of the material, which will, in turn, help optimize material design and textile structural configurations for coated fabrics under complex loading conditions. As a critical indicator of the serviceability of a fabric, tearing strength is rigorously examined when estimating the useful life of the fabric. Although there are simple ways to measure such strength, theoretical prediction and modelling continue to be difficult, due to the many variables that contribute to the complicated mechanisms involved in the tearing process [16-19]. Examples include the development of a del-zone in a tongue tear test (see figure 1). The del-zone is a delta-shaped opening composed of the stretched part of the fabric that bridges the gap between the two tongues. Additionally, it serves to sustain the tearing load at the crack front, to prevent the remarkable yarn movement, slippage and even jamming during fabric tearing, to list just a few. 
These are problems that have attracted much attention in the past 50 years [16-19]. However, for a description of the tearing behaviour with acceptable accuracy, no satisfactory model is presently available, while current research work aimed at developing such models has been mainly experimental and semi-empirical [16-19].

As to the tearing behaviour of coated fabrics, although coating and its penetration prevent yarn slippage during fabric tearing so as to ease the difficulty, the fabric/coating interphase is still viewed as a challenge to any theoretical solution.

As a new attempt at solving the problem, this work represents efforts to apply a stochastic approach, the Ising model aided by the Monte Carlo simulation, to the modelling process. The Ising model was intended, at first, to study the phenomenon of ferromagnetic phase transition [20], but has since become one of the most frequently used models in statistical mechanics for studying systems consisting of interactive subsystems, each in interchangeable states [21-24].

In our analysis, a coated fabric is treated as a system made up of such subsystems as fabric substrate and coating agent that interact with each other through adhesion. The tearing failure of the entire system is the cumulative result of its subsystems changing from bonded states to broken states. These changes are driven by the system energy difference after and before the states change. To be specific, every step in the tearing process corresponds to an energy expression (the Hamiltonian) of the system, and this energy expression incorporates the properties of the fabric substrate and the coating agent, as well as the interactions between them via the interphase. Minimization of the system Hamiltonian yields the most likely new steps for the crack propagation, while the Monte Carlo method is responsible for selecting the one that actually occurs, reflecting the stochastic nature of the process. Allowing the use of a simpler binary algorithm, the Ising model makes for greater efficiency in the simulation.

\section{Description of the model}

In statistical thermodynamics, the macro-characteristics of a system are always a reflection of the interactions and the resulting balance between micro-particles or cells that comprise the system. For a system of $N$ types of subsystems, where the number of subsystems having energy $e_{i}$ is $n_{i}(i=1,2,3, \ldots, N)$, the internal energy of the system is

$$
E=\sum_{i} n_{i} e_{i}
$$

and can be altered by the change of either $n_{i}$ or $e_{i}$

$$
\mathrm{d} E=\sum e_{i} \mathrm{~d} n_{i}+\sum n_{i} \mathrm{~d} e_{i}=\mathrm{d} H+W
$$

where $\mathrm{d} H$ represents change of the energy due to redistribution of the subsystem number $n_{i}$ among energy $e_{i}$. It is also known as the variation of the Hamiltonian $H$, which is the internal energy of a system expressed as the summation of interactions among its constituent subsystems. W represents the system's change of energy due to the change of the subsystems' energies, which can be thermodynamically driven by extrinsic work, such as work of a tear load and friction as in this paper.

\subsection{The system Hamiltonian}

Different methods have been applied in statistical mechanics to express a system internal energy, the Hamiltonian. In this work, the Ising model will be used.

In the original Ising model, a one-dimensional system is divided into a number of lattice cells. Then the Hamiltonian is calculated as the summation of the interactions between each 


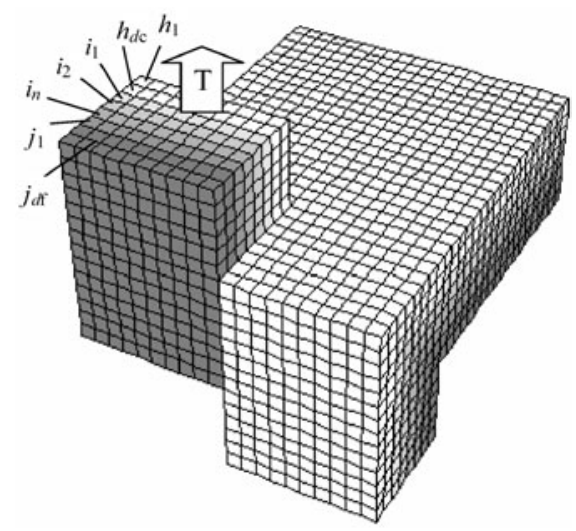

Figure 2. Three-dimensional model for a tongue tear test of a coated fabric with interphase.

pair of the nearest neighbouring cells. The average macroscopic parameters of the system can then be derived from the Hamiltonian via the route of statistical mechanics. The main advantage of the Ising model is that, due to its simple expression, it can be used to describe a complex system made of subsystems with two interchangeable configurations, i.e. by 'digitalizing' the original system into a grid of cells with state 1 or 0 only.

In a tongue tear test, a precut is made into a specimen of the coated fabric. Next, the two tails on both sides of the precut are clamped and pulled apart in opposite directions, as shown in figure 1. To describe the tongue tear test on a coated fabric where an interphase has formed, a discrete three-dimensional model is proposed. Figure 2 shows the portion near the crack front. The coated fabric in a three-dimensional space is divided into a grid of many lattice layers, each composed of a set of cubic cells. The length of a single cubic cell can be chosen arbitrarily and, for the sake of convenience, is made equal to the diameter of a fibre in this work. In the direction of the thickness of the coated fabric, the layers $h_{1}, h_{2}, \ldots, h_{d_{\mathrm{c}}}$ comprise the coating agent and the subscript $d_{\mathrm{c}}$ denotes its thickness, and layers $j_{1}, j_{2}, \ldots, j_{d_{\mathrm{f}}}$ represent the fabric substrate and $d_{\mathrm{f}}$ its thickness. The layers, $i_{1}, i_{2}, \ldots, i_{d_{\mathrm{in}}}$, between them form the interphase with gradient properties and $d_{\text {in }}$ is the thickness of the interphase. It is assumed that properties of a single lattice layer parallel to the fabric plane are constant (an orthotropic system). For a pair of neighbouring cells of the same medium, they are referred to as the connecting cells, while for a pair of neighbouring cells of different media, they are denoted as the contacting cells.

To show the interphase along the crack path in greater detail, a cross-section of one tail in the crack direction is shown in figure 3 . Since the present model is intended to deal with coated fabrics, the relative movement between the yarns in the fabric is neglected because of the coating effect. Therefore, the failure behaviour of the coating and/or fabric cells can be described as a cell that changes from an originally intact state to either an debonding or a broken state.

To represent the possible states of each cell, four variables are introduced:

(i) $M_{i}$, indicating whether a cell $i$ is occupied by the coating agent $\left(M_{i}=1\right)$ or $\operatorname{not}\left(M_{i}=0\right)$;

(ii) $F_{i}$, showing whether a cell $i$ is filled with fabric substrate $\left(F_{i}=1\right)$ or not $\left(F_{i}=0\right)$;

(iii) $f_{i}$, representing whether a cell of fabric substrate is still in a bonded state $\left(f_{i}=1\right)$ or is debonded from the coating agent $\left(f_{i}=0\right)$ in either the contacting cells or in the same cell;

(iv) $m_{i}$, representing whether a cell of coating agent is still in a bonded state $\left(m_{i}=1\right)$ or is debonded from fabric substrate $\left(m_{i}=0\right)$ in either the contacting cells or in the same cell. 


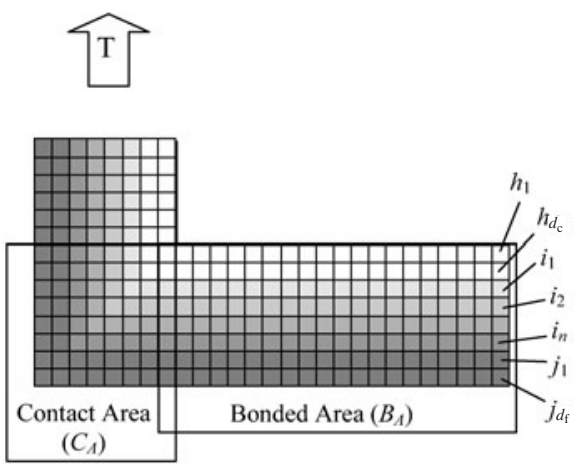

Figure 3. Cross-section of one tail in a tongue tear test.

Table 1. Value of variables for cells in different layers.

\begin{tabular}{lll}
\hline & $M$ & $F$ \\
\hline$h_{1}, \ldots, h_{d_{\mathrm{c}}}$ & 1 & 0 \\
$i_{1}$ & $(n-1) / n$ & $1 / n$ \\
$i_{2}$ & $(n-2) / n$ & $2 / n$ \\
$\ldots$ & \\
$i_{n}\left(n=d_{\text {in }}\right)$ & $1 / n$ & $(n-1) / n$ \\
$j_{1}, \ldots, j_{d_{\mathrm{f}}}$ & 0 & 1 \\
\hline
\end{tabular}

As to the interphase region, a number of fractions are assigned to the variables to represent the mixture of the portions of the two constituents in a given cell, while the summation of the two variables in that cell should be one. Further, it is supposed that a linear decrease of the coating agent occurs in layers $i_{1}, i_{2}, \ldots, i_{d_{\text {in }}}$, as shown in table 1 .

Energy of the system, the Hamiltonian, should be the summation of the energy of each single cell, which in turn is the summation of the interactions between the cell and its connecting or contacting neighbours, as

$$
\begin{gathered}
H=\sum_{i}^{d_{\mathrm{f}}+d_{\mathrm{c}}+d_{\text {in }}} H_{i}=-A \sum_{i}^{d_{\mathrm{f}}+d_{\mathrm{c}}+d_{\text {in }}} \sum_{j}^{\mathrm{cn}} F_{i} F_{j}-B \sum_{i}^{d_{\mathrm{f}}+d_{\mathrm{c}}+d_{\text {in }}} \sum_{j}^{\mathrm{cn}} M_{i} M_{j} \\
-J \sum_{i}^{d_{\mathrm{f}}+d_{\mathrm{c}}+d_{\text {in }}}\left(f_{i} m_{i}+\sum_{j}^{\mathrm{ct}}\left(f_{i} m_{j}+m_{i} f_{j}\right)\right)
\end{gathered}
$$

This equation takes into account all three types of interactions:

(i) Cohesive interaction between the connecting fabric cells, as shown in the first term on the right-hand side of the equation, where $d_{\mathrm{f}}+d_{\mathrm{c}}+d_{\text {in }}$ indicates the sum of the Hamiltonian for cells in all three types of layers including the fabric substrate, the coating agent and the interphase, $A$ represents the unit cohesion energy of the fabric substrate, and cn, summation of $F$ value over all cells in direct connection with cell $i$,

(ii) Cohesive interactions between connecting coating agent cells, as shown in the second term, where $B$ corresponds to the unit cohesion energy of coating agent, and cn denotes summation of $M$ values over all cells in direct connection with cell $i$,

(iii) Adhesive interaction between coating and fabric cells in contact, shown as the terms in parentheses, where $J$ reflects the unit adhesive energy between the fabric substrate and the coating agent, $f_{i} m_{j}$ denotes the adhesive interaction of the fabric substrate with the contacting coating agent in the same cell (for $i=j$ ) and in neighbouring cells (for $i \neq j$ ). 

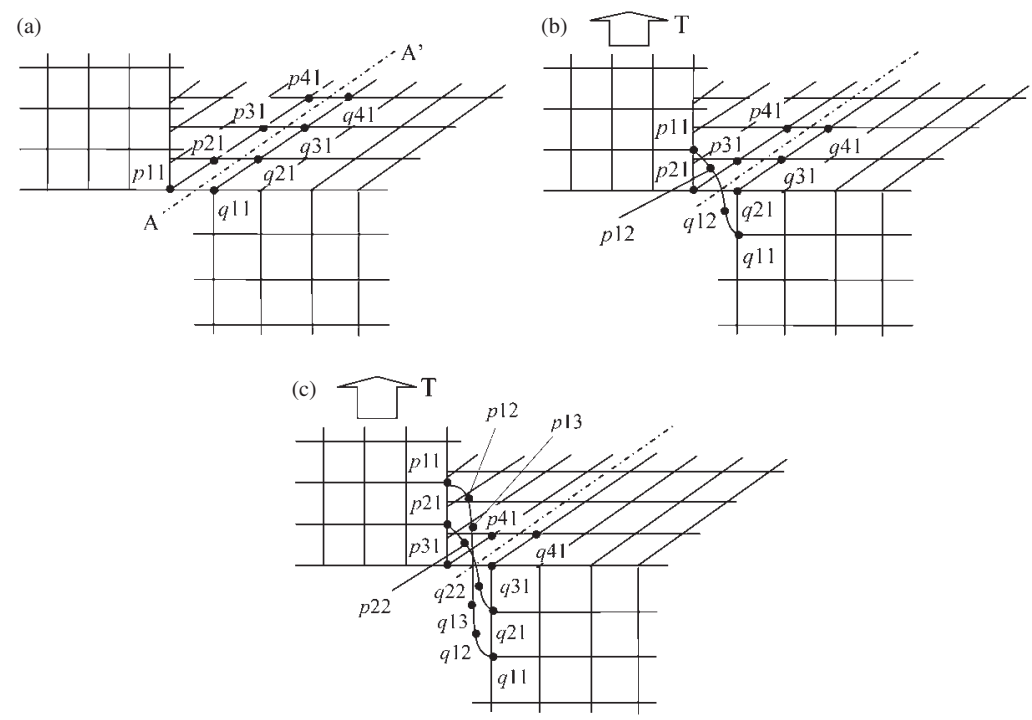

Figure 4. Del-zone at the crack front in a tongue tear test.

\subsection{Work done to the system by external forces}

Upon loading, the material system will change its Hamiltonian from $H_{1}$ to $H_{2}$, and the total energy change $\Delta E$ should also take into account the work done by external forces (tear force and friction resistance) as

$$
\Delta E=H_{2}-H_{1}-W_{\mathrm{T}}+W_{\mathrm{F}}
$$

where $W_{\mathrm{T}}$ denotes work done by tear force and $W_{\mathrm{F}}$ by friction resistance.

$W_{\mathrm{T}}$ is the product of tear force $T$ and displacement of the tail in each step, chosen to be equal to the length of a single cell. Work done by friction equals the product of frictional energy per cell $F_{r}$, and the number of cells in the friction area $F_{\mathrm{A}}$ (see figure 3 ), which can be expressed as:

$$
F_{\mathrm{A}}=C_{\mathrm{A}}-\left(C_{\mathrm{A}} \cap B_{\mathrm{A}}\right)
$$

where $C_{\mathrm{A}}$ represents the area where the two tails contact each other, and $B_{\mathrm{A}}$ denotes the area where the two tails are still bonded together. In other words, friction area $F_{\mathrm{A}}$ is defined as the area at the crack front that has broken from, but is still in contact with, the neighbouring cells.

By calculating the total energy change $\Delta E$ in equation (4) for all possible changes of state in the system, we can, by referring to the $\Delta E$ value, determine the very single change that is most likely to occur.

\section{Monte Carlo simulation}

The stochastic nature of the system behaviour will be accounted for by means of the Monte Carlo simulation via the route of statistical mechanics [21].

In the present model, it is assumed that formation of the del-zone, which bridges the gap between the two tails at the crack front, is caused only by stretching and breakage of the coated fabric. Yarn mobility is non-existent due to the binding effect of the coating agent over the yarns. The development process of the del-zone is shown in figure 4 . To provide a clearer view 
of the process, only one layer of the coated fabric is shown. Each cell in this layer is mapped into a point within the cell so as to effectively represent the media in that cell. It follows that the exact geometric shape of a cell is immaterial in this model.

To begin with, a precut is made into the middle of the coated fabric, and the two tails of the sample, as shown in figure 4(a), are secured in grips so that they will move in opposite directions during tests. Assuming that the crack will propagate along the precut direction $\mathrm{AA}^{\prime}$, and the del-zone will develop symmetrically about the $\mathrm{AA}^{\prime}$ axis, the cells will also move symmetrically about $\mathrm{AA}^{\prime}$ in opposite directions in the contact area and its vicinity. As a result, analysis can be done concerning only half of the del-zone, and a matrix $\boldsymbol{D}(t)=(p x y)$ can be used to describe and trace the evolution of this half del-zone, with pxy representing a cell at position $x$ along the direction $\mathrm{AA}^{\prime}$ and position $y$ along the stretched yarn.

Initially, there is a single cell $p 11$ in the left del-zone

$$
\boldsymbol{D}(0)=p 11=1
$$

The following steps describe how matrix $\boldsymbol{D}(t)$ evolves:

Step 1. When a tear load, $T$, is applied to one tail of the sample, while the other tail is fixed, the pair $p 11$ and $q 11$ move in opposite directions, stretching the part of the sample between them. Focusing on the left side and reflecting the mass conservation during the stretching, a new cell $p 12$ emerges in the left del-zone, as shown in figure $4(b)$, and the media in cell $p 11$ before stretching is halved into cell $p 11$ and $p 12$, after the stretching. But cell $p 21$, which comes right behind $p 11$ along direction $\mathrm{AA}^{\prime}$, remains intact. Thus, the evaluation matrix becomes

$$
\boldsymbol{D}(1)=\left(\begin{array}{cc}
p 11 & p 12 \\
p 21 & 0
\end{array}\right)=\left(\begin{array}{cc}
\frac{1}{2} & \frac{1}{2} \\
1 & 0
\end{array}\right)
$$

During such a process, the increase in internal energy of the system, as can be calculated from equation (3), is gained due to the work of the tear load by stretching the cells (occupied by the fabric substrate and/or the coating agent) as the elastic (strain) energy stored in the system.

Step 2. To continue loading of the left tail, the del-zone is further stretched, and more new cells appear, as shown in figure $4(c)$, and

$$
\boldsymbol{D}(2)=\left(\begin{array}{ccc}
p 11 & p 12 & p 13 \\
p 21 & p 22 & 0 \\
p 31 & 0 & 0
\end{array}\right)=\left(\begin{array}{ccc}
\frac{1}{3} & \frac{1}{3} & \frac{1}{3} \\
\frac{1}{2} & \frac{1}{2} & 0 \\
1 & 0 & 0
\end{array}\right)
$$

so that, for Step $k-1$.

$$
\begin{aligned}
\boldsymbol{D}(k-1)= & \left(\begin{array}{cccc}
p 11 & p 12 & \cdots & p 1 k \\
p 21 & p 22 & \cdots & 0 \\
\vdots & & & \\
p k 1 & 0 & \cdots & 0
\end{array}\right)=\left(\begin{array}{cccc}
\frac{1}{k} & \frac{1}{k} & \cdots & \frac{1}{k} \\
\frac{1}{(k-1)} & \frac{1}{(k-1)} & \cdots & 0 \\
\vdots & & & \\
1 & 0 & \cdots & 0
\end{array}\right) \\
& =\left(\begin{array}{ll}
\frac{\boldsymbol{E}_{1 \times(k-1)}}{k} & \frac{1}{k} \\
\boldsymbol{D}(k-2) & 0
\end{array}\right)
\end{aligned}
$$

where $\boldsymbol{E}_{1 \times(k-1)}=(1,1, \ldots, 1)_{1 \times(k-1)},(k \geqslant 2)$. 
Let $l$ denote the stretching limit (breaking elongation) of the media in a cell, then at step $k$,

$$
\boldsymbol{D}(k)=\left\{\begin{array}{cc}
\left(\begin{array}{cc}
0 & 0 \\
\boldsymbol{D}(k-2) & 0
\end{array}\right)=\boldsymbol{D}(k-2), & k \geqslant l \\
\left(\begin{array}{cc}
\frac{\boldsymbol{E}_{1 \times k}}{(k+1)} & \frac{1}{(k+1)} \\
\boldsymbol{D}(k-1) & 0
\end{array}\right), & k<l
\end{array}\right.
$$

where $\boldsymbol{E}_{1 \times k}=(1,1, \ldots, 1)_{1 \times k}$.

Equation (10) shows that, when cells at the front of the del-zone fail at stretching limit $l$, elements in the first row of matrix $\boldsymbol{D}$ turn into zeros. Otherwise, the stretching process of the del-zone continues.

During this process, variables $M, m$ and $F, f$ for a cell in the del-zone evolve with matrix $\boldsymbol{D}$ and can be updated as:

$$
V_{x y}=\left(V_{0}\right)_{x 1} \cdot(\boldsymbol{D}(k))_{x y}, \quad(V=M, m, F, f)
$$

where $\left(V_{0}\right)_{x 1}$ is the initial value for $V_{x 1}$ (value of variable $V$ for cell $p x 1$ ), from which all the stretched cells, $p x 2, p x 3, \ldots, p x y, \ldots$, are evolved, and $(\boldsymbol{D}(k))_{x y}$ denotes the element in row $x$ and column $y$ of the evolvement matrix $\boldsymbol{D}$ at step $k$.

For each step described above, the energy difference can be calculated by equation (4). With the total energy difference for an arbitrary cell $i$, the probability $W$ for a change of the system from a state with energy $E_{1}$ to one with energy $E_{2}$ is calculated according to the Metropolis function [21]

$$
W\left(E_{2} \mid E_{1}\right)=\min \left[1, \exp \left(\frac{-\Delta E}{\beta}\right)\right], \quad(0<W \leqslant 1)
$$

where $W=1$ when

$$
\Delta E=E_{2}-E_{1} \leqslant 0, \quad \text { or } \quad E_{2} \leqslant E_{1}
$$

that is, a change from a higher energy state $E_{1}$ to a lower one $E_{2}$ is always accepted. Otherwise, $0<W<1$ when

$$
E_{2}>E_{1}
$$

In this case, a number $r$ uniformly distributed between 0 and 1 is randomly generated, and the system's change of state due to thermal perturbation takes place when $r<W$. More frequently, however, in the case where the internal energy difference $\Delta H>0$, change of state is quite unlikely to happen spontaneously in the system, i.e. tearing of the fabric specimen will not take place without a certain amount of work from an external force to make $\Delta E<0$, as indicated in equation (4).

$\beta$ in equation (12) is a thermodynamic constant, reflecting the effect of thermal perturbation, and is proportional to the absolute temperature. For the simulation presented in this study, $\beta$ is consistently given a rather small value (i.e. 0.008), because at room temperature where tongue tear tests are usually carried out, contribution of thermal energy to the system due to thermal fluctuations is negligible. Nonetheless, involvement of temperature is a significant factor in enabling this model to predict effect of temperature changes.

The tear load $T$, increasing from an initial value of zero until adequate for a cell to break/elongate, is determined using an iterative algorithm: $T$ will increase steadily by a small increment of $\mathrm{d} T(=1)$ in each iteration until cell breaking/elongating occurs.

The above process works well either with cells containing only one phase (coating agent or fabric substrate) or with cells in the interphase where breaking elongations happen to be identical (as in fabric substrate and coating agent). However, in more realistic cases where 
elongations of the fabric substrate and the coating agent are different, some adaptation is made to determine the interphase behaviour, as described in the following.

When elongation of an interphase cell $i$ reaches the lower limit of the two, say, that of the coating agent, it may lead to two possible modes of failure:

Failure mode I (FM I). Debonding occurs between the fabric substrate and the coating agent within the same cell $i$ so that $f_{i}=0, m_{i}=0$, with concomitant breaking of the coating agent within the cell so that $M_{i}=0$, while the fabric substrate remains unbroken for further elongation.

Failure mode II (FM II). The fabric substrate and the coating agent in a cell break simultaneously, and the fabric breaks before reaching its breaking elongation because of such factors as stress concentration and dynamic impact due to failure of the coating agent, rendering $M_{i}=F_{i}=m_{i}=f_{i}=0$.

For the interphase, both failure modes are possible during the test. For cells failing in FM I, additional interfacial energy stored in the system will be consumed during the debonding process between the fabric substrate and the coating agent (absent in FM II), so more tear load is required to cause the failure.

In contrast, for cells failing in FM II, the fabric substrate will break before reaching its own stretching limit (absent in FM I) due to stress concentration and dynamic impact caused by failure of the coating agent, thus less load is required.

The actual outcome is again determined by the energy difference, that is, the process that requires less energy input $(\Delta E)$ will most likely occur. In other words, it is the failure mode requiring less external work that will most likely be the outcome. This hypothesis of two failure modes is in fact inspired by our prediction made during the trials, and will be verified in our predictions that are to follow. The complete algorithm of the Monte Carlo simulation is shown in the flow chart in figure 5.

\section{Parametric study}

\subsection{Relationship between the system parameters}

This is a computer simulation of the tear process of a coated fabric. As can be seen from equation (3) and the discussion about failure modes, six parameters are involved in determining the tear behaviour:

(1) Fabric cohesion coefficient $A$, unit cohesion energy between two connecting fabric cells, reflecting the fabric strength.

(2) Coating agent cohesion coefficient $B$, unit cohesion energy between two connecting coating agent cells, corresponding to the strength of the coating agent.

(3) Interfacial adhesive coefficient $J$, unit adhesive interaction energy either between a pair of contacting fabric and coating agent cells, or between the fabric substrate and the coating agent in the same cell, representing the interfacial bonding strength.

(4) $F_{r}$, the friction energy, denoting the friction work exerted during the tear process.

(5) Interphase thickness $d_{\text {in }}$, fabric substrate thickness $d_{\mathrm{f}}$ and coating agent thickness $d_{\mathrm{c}}$.

(6) Breaking elongation $l$, written as $l_{\mathrm{c}}$ and $l_{\mathrm{f}}$, respectively, for the coating agent and the fabric substrate, when the breaking elongation of the coating agent is different from that of the fabric substrate. 


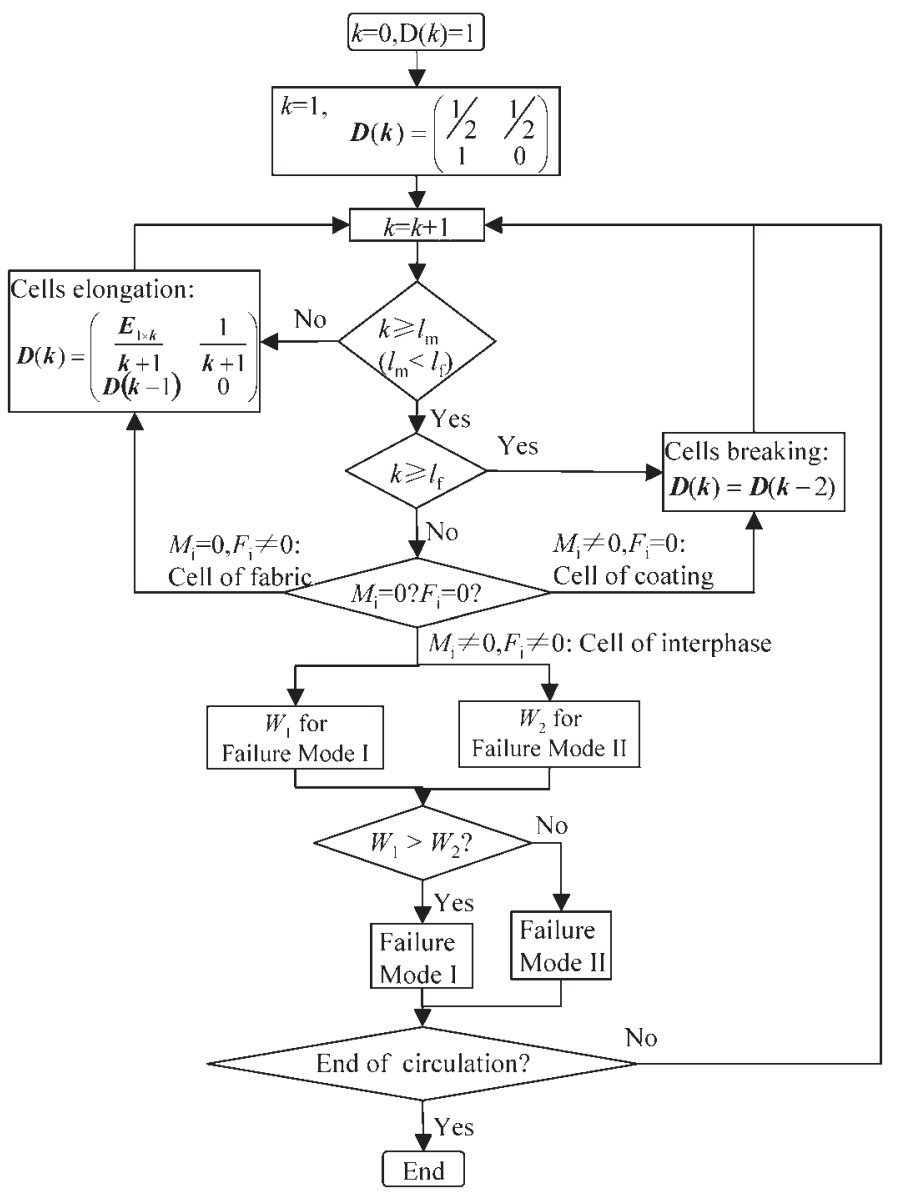

Figure 5. The flow chart of the simulation.

As these parameters correspond to the characteristics of materials involved, parametric studies of the tear process can be carried out by adjusting values of these coefficients so as to derive their corresponding effects on the behaviour of the system.

So, for a given parametric study, we have to vary one of the parameters while keeping the rest constant. Questions then arise about the possible interconnections among these parameters. If they are closely related, when we change the value of one parameter in question, those supposed to remain constant will change as well. In other words, a meaningful parametric study will not be possible unless all the parameters in the model are independent of, or at least very weakly dependent on, each other.

Usually, the interfacial adhesion or bonding strength $J$ can be altered by various surface treatments with little influence on the strength of either fabric or coating agent in the system. Also, there is little if any connection between strengths of the fabric substrate, $A$, and that of the coating agent, $B$. Next, the interphase thickness $d_{\text {in }}$ is determined by the depth of coating penetration, which is affected mostly by fabric construction and manufacturing conditions, and therefore has little to do with other parameters in the model. Likewise, the breaking elongation of the fabric or the coating agent in the del-zone, an intrinsic property of materials, should also be treated as independent. Although mixing both coating agent and fabric substrate does 


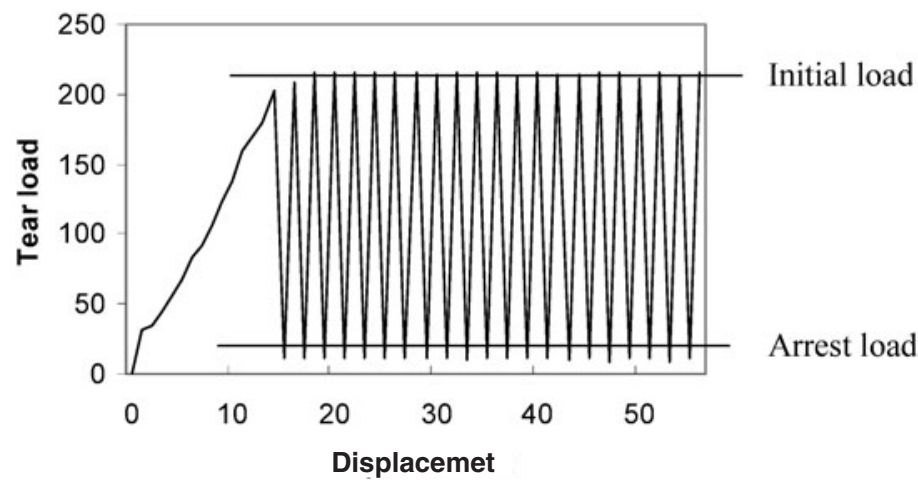

Figure 6. A typical curve predicted of the tear load vs displacement, where $A=500, B=300$, $J=150, F_{r}=50, l_{\mathrm{f}}=4, l_{\mathrm{c}}=2, d_{\mathrm{f}}=8, d_{\mathrm{c}}=2, d_{\mathrm{in}}=2$.

have important effect on the tear behaviour of the interphase in the del-zone, that is done by altering the failure modes of interphase cells as described in the last section, instead of altering the intrinsic properties of materials. This point will be further elucidated in the later sections. Furthermore, although friction takes place between the fabric substrate and the coating agent, it does not necessarily indicate a dependence of $F_{r}$ on either $A$ or $B$, which after all represent only strengths of materials. As for the relationship between $F_{r}$ and $J$, they should be viewed as mutually exclusive, because friction occurs only between contacting cells at the crack that have debonded already from each other.

For generality, exact units and values of $A, B, J$ and $F_{r}$ can be determined by calibrating against actual experimental tests, which will be carried out in our future work. In this paper, all these physical parameters are presented in assumed values and all physical parameters are in relative dimensionless terms.

For the entire tear process of a coated fabric, a typical curve (see figure 6) of tear load applied vs displacement is predicted with the present model; there is at the beginning an increase in the applied load, which continues until the del-zone is fully developed. In the rest of the tear process, the del-zone advances as the fabric or yarn cells entering the crack front are fully stretched and broken in turns. This loading and breaking of continuously entering yarns accounts for the zigzag shape of the curve as expected. Loads applied right before and after the breaking are sometimes called, respectively, the initial load $\left(T_{\mathrm{I}}\right)$ and arrest load $\left(T_{\mathrm{A}}\right)$, as shown in figure 6 . Usually, the former is taken as the mean of the maxima, representing the load required for tear propagation, while the latter the mean of the minima in the curve, i.e. load arresting the crack propagation. The trend of the curve agrees qualitatively with results reported in existing literatures $[18,19]$.

\subsection{The parametric study}

4.2.1. Effect of fabric strength A on tear behaviour. For several sets of parameters with different values of $A$ and the rest kept unchanged, the predicted tear load range $T_{\mathrm{I}} \& T_{\mathrm{A}}$ vs $A$ is shown in figure 7 , to the effect that, with the increase of fabric strength $A$, the initial tear load $T_{\mathrm{I}}$ increases almost in a linear manner, reflecting the fact that a greater load is needed to break a stronger fabric, while the arrest load $T_{\mathrm{A}}$ remains almost constant to retain the crack.

It is worth noting that, by referring to the mechanism discussed in section 3 , this figure helps predict the change of failure mode, and that only the failure mode requiring less external work will be the one that likely takes place. In addition, the results of the simulation show that, 


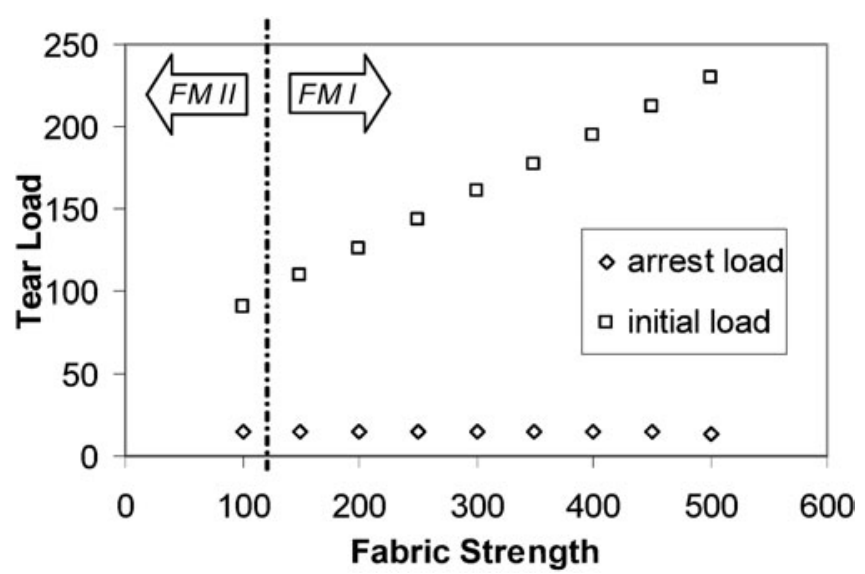

Figure 7. The effect of fabric strength on the tear load, where $A=x, B=300, J=150, F_{r}=50$, $l_{\mathrm{f}}=4, l_{\mathrm{c}}=2, d_{\mathrm{f}}=8, d_{\mathrm{c}}=2, d_{\mathrm{in}}=2$.

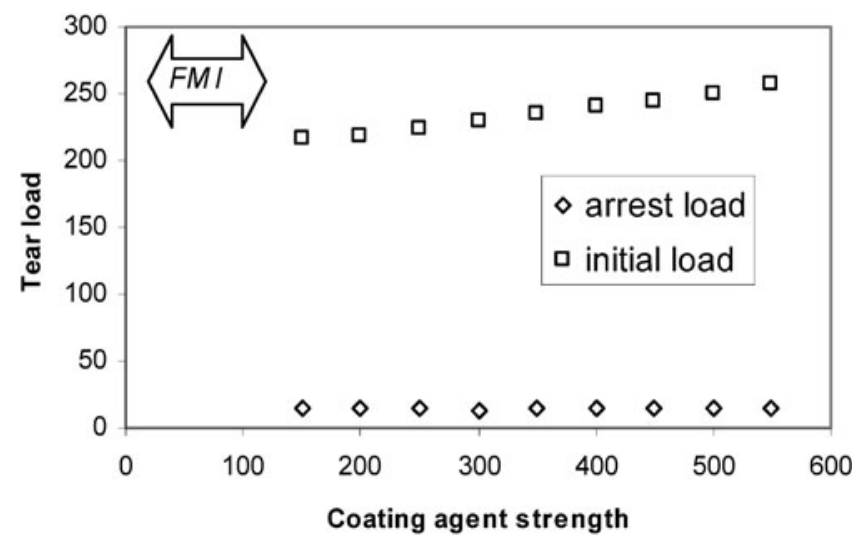

Figure 8. The effect of coating agent strength on the tear load, where $A=500, B=x, J=150$, $F_{r}=50, l_{\mathrm{f}}=4, l_{\mathrm{c}}=2, d_{\mathrm{f}}=8, d_{\mathrm{c}}=2, d_{\text {in }}=2$.

in most cases, failure mode FM I occurs in the interphase cells in the del-zone. On the other hand, when the fabric strength is smaller than the interfacial strength (i.e. when $A<J=150$ in figure 7), FM II will occur in the interphase cells, where the fabric substrate breaks along with the coating agent prior to debonding. For the rest of the cases, where $A>J$, there is always debonding between the mixed fabric substrate and coating agent, so that they will then break, respectively, at their own breaking elongations.

4.2.2. Effect of the coating agent strength $B$ on tear behaviour. The tear behaviour for different values of $B$ is shown in figure 8 . It is found that increase of the strength of coating agent leads to a certain but less marked increase in the required tear load $T_{\mathrm{I}}$, as compared to the case of fabric strength. This is understandable, as the fraction of the coating agent in the whole system is small $\left(d_{\mathrm{c}} / d_{\mathrm{f}}=\frac{1}{4}\right)$. In all cases, then, FM I takes place in the interphase cells; this helps ensure that debonding between the fabric substrate and the coating agent in the del-zone will always occur, and the two media will then break separately at their own breaking elongations. 


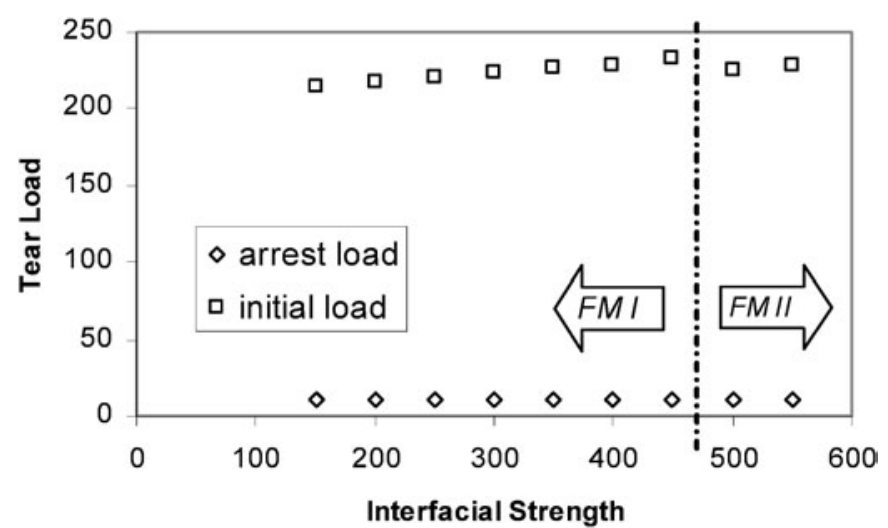

Figure 9. The effect of interfacial strength on the tear load, where $A=500, B=300, J=x$, $F_{r}=50, l_{\mathrm{f}}=4, l_{\mathrm{c}}=2, d_{\mathrm{f}}=8, d_{\mathrm{c}}=2, d_{\text {in }}=2$.

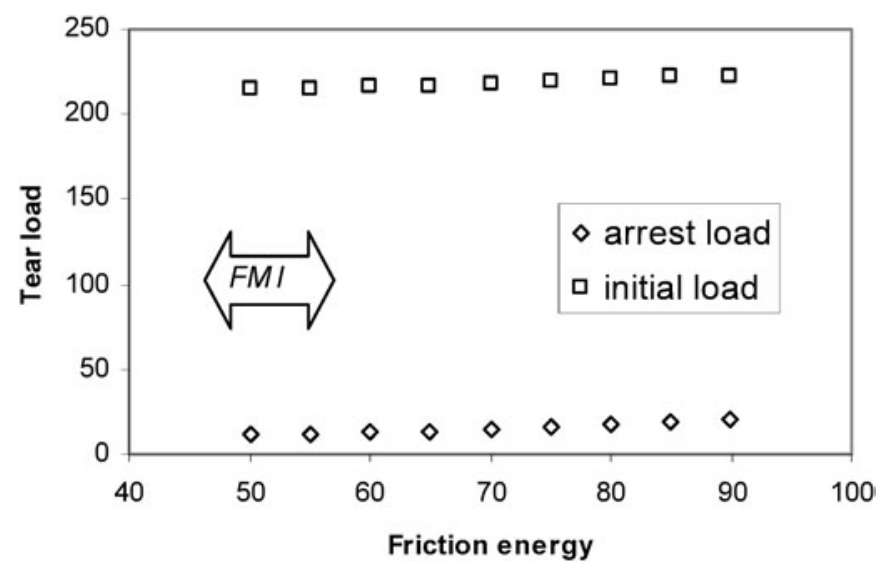

Figure 10. The effect of friction energy on the tear load, where $A=500, B=300, J=150$, $F_{r}=x, l_{\mathrm{f}}=4, l_{\mathrm{c}}=2, d_{\mathrm{f}}=8, d_{\mathrm{c}}=2, d_{\text {in }}=2$.

4.2.3. Effect of interfacial strength $J$ on tear behaviour. When adjusting values of $J$ only, prediction of the tear loads can be made as shown in figure 9. It appears that, initially, tear load $T_{\mathrm{I}}$ increases with the interfacial strength between the fabric substrate and the coating agent. But it is only until the interfacial strength reaches a very high value that failure of interphase cells in the del-zone will change from FM I to FM II. At this point, a slight drop is seen in the tear strength, because, for the fabric substrate to break in FM II along with the coating agent before its own stretching limit is reached, less energy is required, as mentioned previously.

4.2.4. Effect of friction energy $F_{r}$ on tear behaviour. By using different values for $F_{r}$ while keeping other parameters constant, there will be simulated tear loads as shown in figure 10. It indicates that increasing the friction energy will slightly raise the arrest load $T_{\mathrm{A}}$, as the friction will prevent the magnitude from dropping from $T_{\mathrm{I}}$ ever since the breakage begins. Friction will also the raise the initial load $T_{\mathrm{I}}$, as expected.

4.2.5. Effect of breaking elongation on tear behaviour. To study the effect of tensile breaking elongation of the fabric substrate on tear behaviour, tear loads vs fabric breaking elongation $l_{\mathrm{f}}$ 


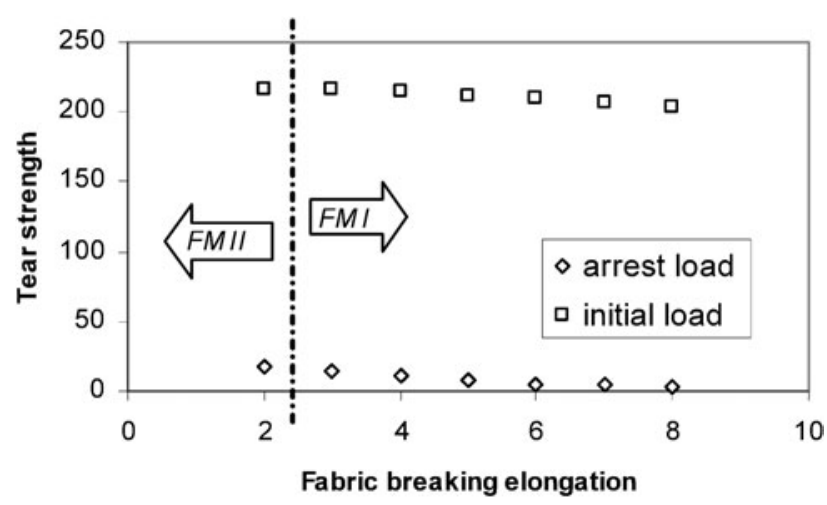

Figure 11. The effect of fabric breaking elongation on the tear load, where $A=500, B=300$, $J=150, F_{r}=50, l_{\mathrm{f}}=x, l_{\mathrm{c}}=2, d_{\mathrm{f}}=8, d_{\mathrm{c}}=2, d_{\mathrm{in}}=2$.

Table 2. Parameter table.

\begin{tabular}{lrllll}
\hline & & & \multicolumn{3}{c}{$d_{\mathrm{d}}^{\mathrm{a}}\left(d_{\mathrm{d}} / d_{\text {in }}\right)$} \\
\cline { 3 - 6 }$d_{\text {in }}=x$ & $d_{\mathrm{f}}$ & $d_{\mathrm{c}}$ & $J=150$ & $J=350$ & $J=550$ \\
\hline 0 & 10 & 4 & 0 & 0 & 0 \\
2 & 9 & 3 & $1(50 \%)$ & $1(50 \%)$ & 0 \\
4 & 8 & 2 & $2(50 \%)$ & $1(25 \%)$ & $1(25 \%)$ \\
6 & 7 & 1 & $3(50 \%)$ & $2(33 \%)$ & $2(33 \%)$ \\
8 & 6 & 0 & $4(50 \%)$ & $3(37 \%)$ & $2(25 \%)$ \\
\hline
\end{tabular}

${ }^{a} d_{\mathrm{d}}$ : number of interphase layers fails in FM I.

is shown in figure 11 . The effect of $l_{\mathrm{c}}$ will not be discussed here, because it has a similar but less significant effect on the system behaviour due to the fact that fraction of the coating agent is small.

It can be said that, with the increase of breaking elongation of fabric substrate, initial load $T_{\mathrm{I}}$ increases slightly at first, as the failure mode of interphase cells in the del-zone changes from FM II to FM I. Then, tear strength decreases with further increase of fabric breaking elongation. This reveals the importance of the ratio of the areas involved as described in equation (5). A greater fabric breaking elongation results in a larger bonded area $B_{\mathrm{A}}$ between the two tails at the crack front, thus in turn reducing the friction area $F_{\mathrm{A}}$, which has been defined in equation (5) as the area at the crack that has broken from, but is still in contact with its neighbouring cells.

4.2.6. Effect of interphase thickness on tear behaviour. When discussing the effect of interphase thickness $d_{\text {in }}$, the situation grows still more complicated, as there is a combination of FM I and FM II in most cases. Here, $d_{\mathrm{d}}$ is introduced as the number of interphase layers that fail in FM I. Values predicted for $d_{\mathrm{d}}$, and for its fraction in the interphase, $d_{\mathrm{d}} / d_{\text {in }}$, are shown in table 2.

To show the combination and competition of FM I and FM II during the tear process, for three systems where interfacial strength $J$ is different, their curves of initial load $T_{\mathrm{I}}$ vs interphase thickness $d_{\text {in }}$ are shown in figure 12 .

In this figure, the solid symbol represents the tear behaviour of an uncoated fabric $\left(d_{\text {in }}=0\right)$, i.e. the fabric substrate. The total thickness $\left(d_{\text {in }}+d_{\mathrm{f}}+d_{\mathrm{c}}\right)$ and the relative fractions of the fabric substrate and coating agent in each of the four coated fabrics are made constant, as shown in 


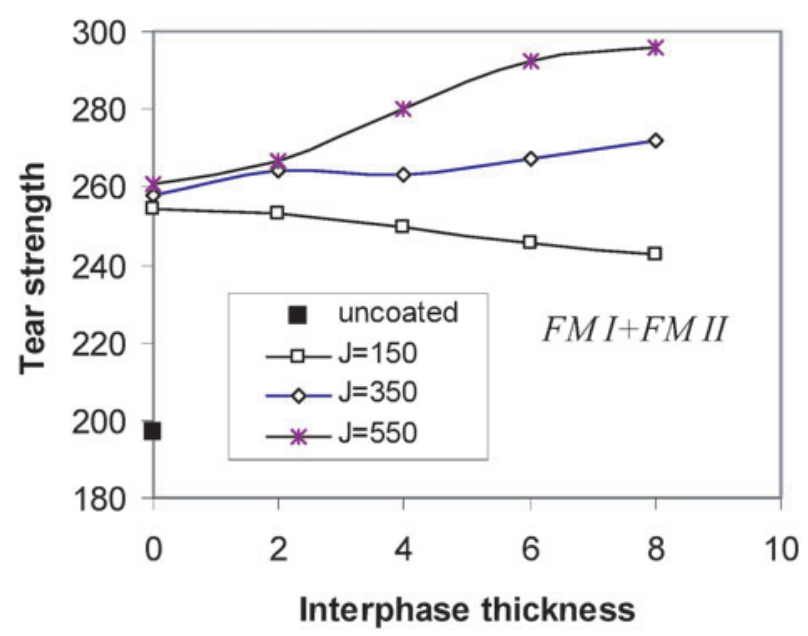

Figure 12. Initial load vs interphase thickness for three systems with different interfacial strengths, where $A=500, B=300, F_{r}=50, l_{\mathrm{f}}=4, l_{\mathrm{c}}=2, d_{\mathrm{f}}, d_{\mathrm{c}}, d_{\text {in }}$ and $J$ listed in table 2 .

table 2, so as to make the results comparable; with increased $d_{\text {in }}$, that is, a deeper penetration of coating agent into fabric substrate, $d_{\mathrm{f}}$ and $d_{\mathrm{c}}$ will both decrease, because the total thickness of the system remains constant.

With a deeper penetration of the coating agent into the fabric substrate, two possible results are seen. First, a deeper penetration of the coating agent into the fabric substrate yields a larger interfacial area between them. This means that more interfacial energy has been stored in the system, requiring higher tear load to cause failure for those cells that will fail in FM I.

Second, a larger interfacial area between the fabric substrate and the coating agent also means that a larger portion of the fabric substrate in the interphase will break, before reaching its own breaking elongation, along with the coating agent. Therefore, less tear load will be required to cause failure for those cells that will fail in FM II.

Since these competing failure modes take effect at the same time, it is the prevailing mode that determines the ultimate tear behaviour of the system.

As shown in figure 12, when the interfacial strength is small $(J=150)$, influence of FM II will dominate the process. Consequently, there will be a decrease in tear strength with increasing interphase thickness.

Then, with rising interfacial strength, influence of FM I tends to dominate. Obviously, the higher the interfacial strength, the higher the interfacial energy stored in the system, even though this would mean a smaller likelihood of debonding (FM I) in the interphase. But this also means that there is a higher probability for the fabric substrate to break prematurely. As shown in figure 12, when $J$ reaches 350 , tear strength rises with interphase thickness, except that there is a drop of $d_{\mathrm{d}} / d_{\text {in }}$ from $50 \%\left(\right.$ when $d_{\text {in }}=2$ ) to $25 \%\left(\right.$ when $d_{\text {in }}=3$ ), which indicates further decreased debonded area, or a larger portion of FM II failure in the interphase.

Otherwise, in the case of $J=550(>A=500)$, effect of the interfacial energy will overwhelm, and the tear strength will increase regularly despite a drop in $d_{\mathrm{d}} / d_{\text {in }}$ from $33 \%$ (when $d_{\text {in }}=6$ ) to $25 \%$ (when $d_{\text {in }}=8$ ).

In summary, the present model is useful because it helps reveal the important effect the interphase has on the tear behaviour of a coated fabric, by means of altering the failure mode of the interphase cells, instead of the intrinsic properties of the materials, as mentioned in section 4.1. These could be significant findings explaining the synergetic effect [25] or the hybrid effect [26] during fracture of a composite system. 
It should be clear by now that first, the Ising model is capable of describing such complicated system as a coated fabric with interphase in a simple binary form, accounting for all the physical mechanisms involved, and depicting the stochastic nature of the behaviour of materials, yet generating robotically informative results. Second, obviously beging able to depict such intricate structure as the del-zone developed in the tear process, this approach proves to be especially useful for explaining the effect of the interaction that occurs at the interphase within heterogeneous materials. This method also manifests itself in such phenomena as the synergy effect and the so-called hybrid effect-a task that has been found difficult to tackle with existing models. Finally, as this work deals with tear behaviour of fabrics with constrained yarn mobility, the model can be further modified to study the tear behaviour of fabrics with considerable yarn movements during fabric tearing, as well as other similar interfacial problems.

Additionally, it is worth adding a few words about other tasks involved when using the Ising model. This includes the determination of all the involving material parameters, which, in theory, can be attained by calibrating the model against properly designed experiments. It takes the same trouble to determine the ranges of the various interactions between cells. In this study, however, interaction occurs only between cells either in contact or in connection with each other; it may not be the case when dealing with interaction between, say, fluid cells.

\section{Conclusions}

A study applying a stochastic approach, the Ising model combined with the Monte Carlo method, is carried out to simulate the tongue tear process for a coated fabric, where the effect of the interphase between the fabric substrate and the coating agent is taken into account. As a result, complicated mechanisms involved are realistically simulated.

Several advantages of the approach are demonstrated in this paper. First, the Ising model is capable of describing such complicated system as a coated fabric with interphase in a simple binary form, accounting for all physical mechanisms involved, and depicting the stochastic nature of the behaviour of materials, yet generating robotically informative results. Second, obviously being able to depict such intricate structure as the del-zone developed in the tear process, this approach proves to be especially useful for explaining the effect of the interaction that occurs at the interphase within heterogeneous materials. This method also manifests itself in such phenomena as the synergy effect and the hybrid effect. These justify the use and demonstrate the power of such an approach in predicting the mechanical characteristics of heterogeneous materials.

Model predictions are followed by a series of parametric studies to investigate influences of important parameters on the tear behaviour. Results are in good accordance with reported outcomes in existing literature, which further validates application of the method.

In this paper, physical parameters and simulated results are expressed in relative and dimensionless terms, which, we believe, can help better demonstrate usefulness of this new approach. But future work may have to involve determination of these parameters by calibrating against actual experimental tests to give a direct relationship between these parameters and real properties of materials.

In this work, constrained yarn mobility within the fabrics is assumed for the tear process in a fibre/matrix system to simplify the problem. Further studies may be performed on failure behaviour with considerable yarn movement in the fabrics so that slippage between the yarns and the jamming effect can be included. Further, it is necessary to tackle the more difficult yet realistic situation where the fibre, matrix and even the bonding properties can be treated as statistically distributed. 


\section{Acknowledgment}

The authors would like to thank the support in part from the National Textile Center Grant No M02-E03 for this study.

\section{References}

[1] Gardner S D, Pittman C U and Hackett R M 1993 Polymeric composite materials incorporating an elastomeric interphase: a mathematical assessment Compos. Sci. Technol. 46 307-18

[2] Low B Y, Gardner S D, Pittman C U and Hackett R M 1994 A micromechanical characterization of graphitefiber/epoxy composites containing a heterogeneous interphase region Compos. Sci. Technol. 52 589-606

[3] Cox H L 1952 The elasticity and strength of paper and other fibrous materials Br. J. Appl. Phys. 3 72-9

[4] Paipetis A, Galiotis C, Liu Y C and Nairn J A 1999 Stress transfer from the matrix to the fiber in a fragmentation test: Raman experiments and analytical modeling J. Compos. Mater. 33 377-99

[5] Wu W, Verpoest I and Varna J 1998 An improved analysis of the stresses in a single fiber fragmentation test, II. Three-phase model Compos. Sci. Technol. 58 41-50

[6] Nairn J A and Liu Y C 1997 Stress transfer through a fragmented, anisotropic fiber through an imperfect interface Int. J. Solids Struct. 34 1255-81

[7] Dong Y S, Nobuo T, Tadashi S and Kazuo N 1996 Approximate analysis of the stress state near the fiber ends of short fiber reinforced composites and the consequent microfracture mechanisms Compos. Part A 27 357-64

[8] Carman G P, Averill R L, Reifsnider K L and Reddy J N 1993 Optimisation of fiber coatings to minimize stress concentrations in composite materials J. Compos. Mater. 27 589-612

[9] Kim P 1999 Stress distributions in three-phase composites Compos. Interface $\mathbf{5} 441-50$

[10] Hayes S A, Lane R and Jones F R 2001 Fiber/matrix stress transfer through a discrete interphase: Part 1. Single-fiber model composites Compos. Part A 32 379-89

[11] Lane R, Hayes S A and Jones F R 2001 Fiber/matrix stress transfer through a discrete interphase: Part 2. High volume fraction system Compos. Sci. Technol. 61 565-78

[12] Lopattananon N, Kettle A P, Tripathi D, Beck A J, Duval F, France R M, Short R D and Jones F R 1999 Interface molecular engineering of carbon-fiber composites Compos. Part A 30 49-57

[13] Gao S L and Mader E 2002 Characterization of interphase nano-scale property variations in glass fiber reinforced polypropylene and epoxy resin composites Compos. Part A 33 559-76

[14] Tripathi D, Chen F and Jones F R 1996 A comprehensive model to predict the stress fields in a single fiber composite J. Compos. Mater. 30 1514-38

[15] Jiang L Y and Wang X D 2002 On the dynamic crack propagation in an interphase with spatially varying elastic properties under in-plane loading Int. J. Fracture 114 225-44

[16] Chen Y, Lloyed D W and Harlock S C 1995 Mechanical characteristics of coated fabrics J. Textile Ins. 86 690-700

[17] Hamkins C P and Backer S 1980 On the mechanisms of tearing in woven Fabrics Textile Res. J. 50 323-7

[18] Scelzo W A, Backer S and Boyce M C 1994 Mechanistic role of yarn and fabric structure in determining tear resistance of woven cloth: Part I. Understanding tongue tear Textile Res. J. 64 291-304

[19] Scelzo W A, Backer S and Boyce M C 1994 Mechanistic role of yarn and fabric structure in determining tear resistance of woven cloth: Part I. Modeling tongue tear Textile Res. J. 64 321-9

[20] Ising E 1925 A contribution to the theory of Ferromagnetism Z. Physik 31253

[21] Yeomans J M 1992 Statistical Mechanics of Phase Transition (New York: Oxford Science)

[22] Zhong W, Ding X and Tang Z L 2001 Modeling and analyzing liquid wetting in fibrous assemblies Textile Res. J. $71762-766$.

[23] Zhong W, Ding X and Tang Z L 2002 Analysis of fluid flow through fibrous structures Textile Res. J. 72 751-5

[24] Lukas D, Glazyrina E and Pan N 1997 Computer simulation of liquid wetting dynamics in fiber structure using Ising's model J. Textile Inst. 88 149-61

[25] Pan N 1995 A detailed examination of the translation efficiency of fiber strength into composite strength J. Reinforced Plast. Compos. $142-28$

[26] Pan N and Postle R 1996 Tensile strengths and the hybrid effects of hybrid fiber composites: a probabilistic approach Trans. R. Soc. Lond. Ser. A 354 1875-97 